\title{
Entre el derecho de los diseños y las patentes: la tecnología ponible*
}

\section{Ana Karin Chávez Valdivia**}

\begin{abstract}
RESUMEN
La Propiedad Intelectual se constituye en uno de los activos más importantes que tienen las empresas. Si bien tradicionalmente ha existido una clara diferencia entre los diseños industriales y las patentes al tiempo que debido a la propia naturaleza del diseño surgió la necesidad de plantear diversas posibilidades de tutela en torno a esta figura, el desarrollo tecnológico podría llevarnos a cuestionar los actuales mecanismos de protección. La tecnología ponible se encuentra en auge e implica una interacción de dos sectores que son aún materia de análisis, discusión y falta de consenso: la moda y la tecnología. Consideramos que podría llegar a producirse la desnaturalización del diseño industrial ante la inminente posibilidad de aceptación de una omisión de la forma con función que sumada a la poca claridad que existe respecto a la tutela de diseños no registrados y carencia normativa en torno a los diseños de tecnología ponible nos conducirian a un obligado análisis sobre la pertinencia de aplicación de los mecanismos legales actuales en el ámbito tecnológico y la necesidad de contar con normativas acordes a las innovaciones tecnológicas existentes.
\end{abstract}

\section{PALABRAS CLAVE}

Diseños industriales, diseños no registrados, patentes, derechos de autor, tecnología ponible, moda.

\begin{abstract}
Intellectual Property is one of the most important assets that companies have. Although traditionally it has existed a clear difference between industrial designs and patents, due to the very nature of the design, there was a need to raise various possibilities to safeguard around this technological figure, whose development could lead us to question the current mechanisms of protection. Wearable technology is booming and involves an interaction of two sectors that are still a matter of analysis, discussion and lack of consensus: fashion and technology. We believe that the denaturalization of industrial design could occur in the face of the imminent possibility of acceptance of an omission of the form with a function that, added to the lack of clarity that exists regarding the protection of unregistered designs and the lack of regulations surrounding the design of wearable technology, that would lead us to a forced analysis on the relevance of application of current legal mechanisms in the technological field and the need to have regulations in line with existing technological innovations.
\end{abstract}

\section{KEYWORDS}

Industrial designs, unregistered designs, patents, copyrights, wearable technology, fashion. 
SUM ARIO

1. Introducción

2. Una mirada hacia la moda

3. Protección jurídica de la moda en el Perú y en la legislación comparada

4. La tecnología ponible (wearable technology)

5. Consideraciones finales

\section{Introducción}

El desarrollo tecnológico ha incidido de diferentes formas en todos los ámbitos de la sociedad y del derecho. Evidentemente, la propiedad intelectual no le ha sido ajena; más aún, se presenta como uno de los campos que mayores desafíos plantea al jurista.

Nos encontramos ante una realidad contradictoria. El vertiginoso desarrollo de una nueva tendencia en la industria de la moda llamada moda funcional se enfrenta al hecho de que el llamado Fashion Law se encuentra en auge en algunos países, pero en muchos otros se carece aún de un marco legal propio para el sector de la tradicional moda convencional.

$\mathrm{Al}$ mismo tiempo, el cambio constante y la vida efímera de la industria de la moda revelan la necesidad de reconocer que las actuales formas de protección contempladas por el derecho tal vez son insuficientes para subsanar algunas deficiencias y ofrecer soluciones certeras a la prometedora relación moda y tecnología dentro de la cual convergen algunos clásicos derechos de la propiedad intelectual.

El presente estudio revisa las principales disposiciones en relación con la normativa peruana y comparada. Se intenta analizar la posible desnaturalización de algunas instituciones jurídicas tradicionales que, en la búsqueda de tutelar determinadas creaciones producto de la innovación tecnológica, parecieran conducirnos a desdibujar las líneas que hasta hace poco separaban los procesos creativos conocidos y sus resultados. Al mismo tiempo, se busca dar a conocer algunas imprecisiones y vacíos en torno a la legislación vigente que revelan la innegable necesidad de contar con mecanismos de tutela orientados a la tecnología ponible. 


\section{Una mirada hacia la moda}

La moda es aquello que pasa de moda, y su representación estadística es la curva de Gauss (función matemática que describe el ciclo completo que incluye la ascensión), el punto máximo de apogeo y luego el declive. ${ }^{1}$

El comienzo de la industria de la moda lo encontramos en París, aproximadamente alrededor del año 1850. Su origen se le atribuye a Charles Frederich Worth, quien tuvo la intención de vestir a las mujeres reuniendo novedad y estilo.

Por otro lado, Salas refiere que, a pesar de que por cientos de años la moda fue observada únicamente como la necesidad básica de vestirse, se convirtió en el lenguaje empleado por hombres y mujeres en distintos momentos de la historia. En este sentido, la industria de la moda ha sido entendida como una manera pasajera de ser o de hacer, pero largamente adoptada y valorizada en un grupo y con alcance cultural. Por lo tanto, se convierte en un concepto transversal que se extiende incluso a las maneras de pensar de una sociedad y no solamente se aplica al ámbito textil y al vestido. Al mismo tiempo, esto involucra una serie de conceptos como la creación, creatividad y novedad, términos todos que se traducen en la llamada innovación. ${ }^{2}$

Adicionalmente, la autora considera que la industria de la moda juega con dos elementos que mutan constantemente: las tendencias y el estilo. Las primeras hacen referencia a las orientaciones colectivas de una época, un país, un grupo o de un creador que se afirman vigorosamente en el mercado. En tanto el segundo elemento es considerado como la manera particular de tratar la materia y las formas con miras a la realización de una obra (de arte). De ahí que se entienda por estilo al conjunto de características de una obra que permiten clasificarla con otras en un conjunto que constituye un tipo estético.

Es así que la presencia de los dos elementos señalados hace posible la producción de la moda a cargo de diseñadores y creadores, y nos sitúa ante la figura de un creador artista. Por su naturaleza, éste debe ser ingenioso e innovador. En cuanto a este último punto, precisa Salas, cuando se habla de innovación no se hace referencia al concepto previsto en materia de patentes, es decir, a la invención entendida como una solución a un problema no resuelto en el estado de la técnica. Más bien, se trata de una innovación desde el punto de vista

\footnotetext{
1 Erner, Guillaume, Sociología de las tendencias, Barcelona, Gustavo Gili, 2013.

2 Salas Pasur, Brenda, "La industria de la moda a la luz de la propiedad intelectual", La Propiedad Inmateria, vol. 17. [Fecha de consulta 1 julio 2017]. Disponible en: http://revistas.uexternado.edu.co/index.php/propin/article/ view/3583/3800
} 
estético, que deberá expresar las fantasías e innovaciones de esta naturaleza en materia de indumentaria. La referencia a una creación de forma estética $u$ ornamental, concluye, corresponde a ese fenómeno.

Sin embargo, debe considerarse que algunas veces una creación dentro del sector de la moda presenta innovaciones técnicas. En ese caso, el carácter estético se conjuga o da lugar a otra dinámica: la de la invención. Acorde con que la creación de la moda sea calificada de innovación técnica, de creación ornamental o de creación artística, responderá a un régimen de protección diferente.

Según las consideraciones acotadas, podemos afirmar que la moda es una manifestación de la cultura de una sociedad en un lugar y tiempo determinado. Por ello, con el transcurrir de los años ha pasado de cumplir un rol únicamente utilitario (necesidad básica de vestirse) a tener una finalidad estética. Actualmente consideramos que se le ha añadido un rol funcional jamás pensado, producto del desarrollo tecnológico; esto es, la moda de tecnología ponible.

Es así que la moda contemporánea, además de comprender indumentaria (entendida tiempo atrás únicamente como prendas de vestir), a la fecha hace referencia a un universo tecnológico heterogéneo que incluye diversos segmentos como relojes, computadoras, joyas, teléfonos, gafas, artículos deportivos, productos que permiten visualizaciones en espacio 3D, entre otros.

En ese orden de ideas, deviene necesario analizar el régimen de protección en el cual se enmarca esta industria creativa, toda vez que ella se orienta hacia dos ángulos: el estético y el funcional.

\section{Protección jurídica de la moda en el Perú y en la legislación comparada}

La moda es una de las industrias más grandes del mundo. Al mismo tiempo, es una de las más antiguas. Su protección siempre ha gravitado en torno a dos ramas de la propiedad intelectual: el derecho de autor y la propiedad industrial.

En lo respecta al primero, se regula toda creación intelectual, personal y original, susceptible de ser divulgada o reproducida en cualquier forma conocida o por conocer. Tales creaciones están protegidas cuando pertenezcan al ámbito literario o artístico, cualquiera que sea su género, forma de expresión, mérito o finalidad. En tanto, la propiedad industrial trata principalmente de la protección de las invenciones, marcas, los diseños industriales, modelos de utilidad, el nombre comercial, entre otros.

Si nos referimos a la relación entre el derecho de autor y el sistema de patentes, por ejemplo, encontramos un común denominador: en ambos casos 
existen aportaciones creadoras. En el primero, una obra literaria o artística; en el segundo, una invención aplicable a la industria.

Sin embargo, existen también diferencias sustanciales entre ambos sistemas, no sólo en lo referente al objeto de protección -es decir, la forma de expresión en el derecho de autor y la solución técnica en el derecho de invención-, sino también en torno al reconocimiento y amplia protección de los derechos morales. Esto es, el tiempo de duración de ambos, la tutela de la obra, aunque permanezca inédita frente a la posible caducidad de la patente por falta de explotación, entre otras.

En ese sentido, uno de los principales problemas en torno a la industria de la moda es identificar la protección que reconoce la propiedad intelectual a cada elemento o instrumento propio de esta industria. Por lo tanto, podría ser que el proceso de transformación de un material textil se proteja a través del sistema de patentes: que la línea de la colección se identificara bajo un signo distintivo o que el impreso de la tela se registrase como diseño industrial.

$\mathrm{Al}$ mismo tiempo, entre el derecho marcario y el derecho de autor existen importantes diferencias. Entre estas podemos mencionar la eficacia distintiva de la marca ante la originalidad en la forma de expresión de las obras literarias o artísticas; el carácter temporal del derecho de autor frente a la posibilidad de que la marca, mediante renovaciones sucesivas, pueda mantenerse con carácter permanente, salvo que se extinga por falta de renovación; la protección de la creación literaria y artística con prescindencia de toda formalidad, ante el registro necesario que aparece en el derecho marcario; o la tutela del derecho de autor sobre la obra, como bien autónomo e independiente, frente a la vinculación de la marca como un producto, empresa o servicio.

Por otro lado, al considerar el aspecto estético dentro del campo del derecho de autor y de la propiedad industrial, identificamos, por una parte, a la obra de arte y la de arte aplicado y, por la otra, a los diseños industriales.

En el Perú, ${ }^{3}$ las creaciones de moda son consideradas como obra de arte aplicado, siempre y cuando cumplan con el requisito de originalidad que la ley establece para tal fin. Es decir, que la creación en cuestión exprese la personalidad del diseñador y no sólo provenga de la naturaleza de las cosas.

Estas obras, tal como los modelos de joyería, orfebrería, bisutería, mueblería, vestidos y decoración, se mueven en el terreno movedizo entre el derecho de invención y el derecho de autor. Lo anterior porque tienen por su forma de expresión una naturaleza artística, pero por su destino, una utilización industrial.

\footnotetext{
3 "Ley sobre el Derecho de Autor", Diario Oficial El Peruano, 24 de abril, 1996.
} 
Es por esta razón que el Convenio de Berna, ${ }^{4}$ en su artículo 2 numeral 7, deja reservada a las legislaciones nacionales la facultad de regularlo concerniente a las obras de arte aplicadas. Incluso disminuye el plazo de protección en comparación con otras obras del ingenio, siempre que no sea inferior de 25 años a partir de su realización. Sin embargo, sobre las obras protegidas únicamente como dibujos y modelos en el país de origen, no puede reclamarse más que la protección especial concedida en este último a los dibujos y modelos en otro país de la Unión. Si tal tutela específica no se concediera en este país, estas obras deben ser tuteladas como artísticas, lo que plantea la posibilidad de la protección acumulada.

A su vez, el artículo 5 quinquies del Convenio de Paris ${ }^{5}$ dispone que los dibujos y modelos industriales estén protegidos en todos los países de la Unión. Sin embargo, nada impide que la tutela hacia determinados diseños, como los aplicables a la indumentaria, esté enmarcada dentro de los derechos de autor.

Por su parte, el Acuerdo sobre los Aspectos de los Derechos de Propiedad Intelectual Relacionados con el Comercio (ADPIC), en el seno de la Organización Mundial del Comercio (омc), dispone que los países miembro tendrán libertad para cumplir con la protección de los dibujos o modelos textiles mediante la legislación sobre dibujos o modelos industriales, o a través de la legislación sobre el derecho de autor. ${ }^{6}$

Como hemos señalado, acorde con nuestra legislación, las creaciones de la moda son consideradas obras de arte aplicado. Estas obras son conceptualizadas como creaciones artísticas con funciones utilitarias o incorporadas a un artículo útil, ya sea una obra de artesanía o una producida a escala industrial.

Sin embargo, no todas las creaciones de la moda reciben esta protección, sino sólo aquellas que constituyan o incorporen una creación artística original, como podría ser un diseño, dibujo o patrón original, en el cual se refleje su impronta o creatividad. En este orden de ideas, por ejemplo, estará protegido por el derecho de autor un vestido cuya forma, particularidades del corte, bordado $\mathrm{u}$ otros, hagan de la pieza resultante algo original. La protección se hace extensible tanto a las prendas de vestir en general como a los llamados accesorios. ${ }^{7}$

\footnotetext{
4 "Convenio de Berna para la protección de las obras literarias y artísticas", Organización Mundial de la Propiedad Intelectual. [Consulta: 2 de julio, 2017]. Disponible en: http://www.wipo.int/treaties/es/text.jsp?file_id=283698 5 "Convenio de Paris para la Protección de la Propiedad Industrial", Organización Mundial de la Propiedad Intelectual. [Consulta: 2 de julio, 2017]. Disponible en: http://www.wipo.int/wipolex/es/treaties/text.jsp?file_id=287557 6 "Acuerdo sobre los aspectos de los Derechos de Propiedad Intelectual relacionados con el comercio", World Trade Organization. [Consulta: 5 de julio, 2017]. Disponible en: https://www.wto.org/spanish/docs_s/legal_s/27-trips.pdf 7 "Ley sobre el Derecho de Autor", Diario Oficial El Peruano, 24 de abril, 1996.
} 
El autor en este tipo de obras es el diseñador. No es necesario que éste sea reconocido, cuente con una gran trayectoria, trabaje en una casa de alta costura o el diseño en cuestión esté hecho a mano a través de un sistema informático. Además, podemos encontrar creaciones consideradas como obras en colaboración y obras por encargo.

Nuestra legislación contempla también la posibilidad de proteger las creaciones de moda mediante las siguientes modalidades:

- Signo distintivo. En este caso, sólo se protegen la marca o logotipo, mas no la prenda de vestir a la cual se aplica, sin perjuicio de que se intente registrar una forma particular como marca tridimensional. Sin embargo, nada impide que la figura o los dibujos del signo distintivo constituyan per se una manifestación artística. En ese caso, puede coexistir una doble protección: como marca y como obra estética.

- Diseño industrial. Al ser considerado como una forma visible que incorporada a un producto utilitario, le da un aspecto o apariencia especial o diferente. Posibilita la protección de obras bidimensionales o tridimensionales que pueden ser incorporadas a las creaciones de moda y que son producidos a escala industrial. Por citar un ejemplo, se protegería el diseño del patrón de Gucci, ${ }^{8}$ pero no el modelo o diseño del artículo de moda en sí mismo. Es decir, una creación de moda no sólo puede estar protegida por el derecho de autor, sino también puede estarlo por el derecho marcario, respecto al signo distintivo registrado que ostente, así como por el diseño industrial que pueda contener o presentar. Estos derechos son independientes uno del otro, tanto en su reconocimiento o validez, como en su alcance, ámbito y nivel de protección.

Indiscutiblemente, entre las disciplinas señaladas se presentan zonas fronterizas que hacen posible la protección de un mismo bien intelectual dentro de cada una de ellas bajo diferentes ópticas y con distinto contenido.

Por otro lado, si revisamos la legislación comparada, encontramos que en la legislación norteamericana las prendas de vestir como tales no se encuentran protegidas por la sección 101 del Copyright Act. ${ }^{9}$ Las fotografías, gráficos y esculturas sí son objeto de protección, siempre que se puedan separar y existir

\footnotetext{
${ }^{8}$ Diseño D359,166 de la uspto (Oficina de Marcas y patentes de los Estados Unidos de Norteamérica). Creado por Ford, Thomas para Guccio Gucci S.p.A

9 "Copyright law of the United States", Copyrigth.gov. [Consulta: 10 de julio, 2017]. Disponible en: https://www. copyright.gov/title17/title17.pdf
} 
de manera independiente del objeto al que se incorporan. Hablamos entonces de la teoría de la separabilidad, en la cual el análisis se reduce a resolver el interrogante relativo a la dicotomía del arte y función: un diseño que es funcional no puede ser ornamental.

En ese orden de ideas, la industria de la moda, y en particular los diseños de moda, aún no encuentra protección expresa vía derecho de autor en los Estados Unidos. ${ }^{10}$ Ello no significa que este tipo de creaciones no sean objeto de protección, ya que encuentra tutela en las figuras tradicionales como las marcas, el Trade Dress ${ }^{11}$ y las patentes de diseño. ${ }^{12}$

La Unión Europea, por su parte, en torno a este tema, buscó establecer un mecanismo uniforme que brindara seguridad jurídica a los diseñadores de moda a través de los dibujos o modelos, ambas figuras contempladas en nuestra legislación como diseño industrial. Para este fin, expidió la Directiva Europea 98/71, acompañada de su reglamento de ejecución. Su efecto fue establecer lineamientos para que los países miembro de la Unión incorporaran esta herramienta a su normatividad interna, manteniendo el principio de la unidad de arte. Es así que la originalidad del sistema radicó en la creación de tres formas de protección:

- El establecimiento del dibujo o modelo comunitario registrado (DMCR) ${ }^{13}$

- El establecimiento de un mecanismo sui generis de protección a través del dibujo y modelo comunitario no registrado (DMCNR) ${ }^{14}$

- La protección acumulativa ${ }^{15}$

\footnotetext{
${ }^{10}$ Debido a que las cortes estadounidenses son claras en afirmar que los objetos que cumplan una función utilitaria no pueden ser tutelados por el ordenamiento jurídico (a diferencia de los accesorios de moda como joyas, estampados y hebillas de pantalones protegidos a través del derecho de autor), se intentó promover la "Innovative Design Protection Act" del 2012. Esto con el fin de reconocer a esta industria vía derechos de autor por un término de tres años, proyecto que no prosperó.

${ }^{11}$ Forma particular de propiedad intelectual que específicamente protege la apariencia o la forma de un producto que el público reconoce que viene de un origen particular. No obstante, es muy difícil adquirir la protección porque requiere de ventas activas, publicidad y promoción, porque debe existir una asociación particular entre la apariencia con la compañía que lo fabrica.

12 "Whoever invents any new, original and ornamental design for an article of manufacture may obtain a patent therefor, subject to the conditions and requirements of this title. The provisions of this title relating to patents for inventions shall apply to patents for designs, except as otherwise provided". Véase "35 U.S. Code § 171 - Patents for designs", Legal Information Institute. [Consulta: 2 de julio, 2017]. Disponible en: https://www.law.cornell.edu/ uscode/text/35/171

${ }^{13}$ Permite amparar aquellas creaciones con periodos de vida largos, es decir, que requieren un instrumento de protección prolongado.

14 Protege aquellas creaciones cuyo periodo de vida es más corto.

${ }^{15}$ Protección mediante el derecho de autor y la propiedad industrial a través del dibujo o modelo.
} 
No obstante, el documento en mención también dejó abierta la posibilidad de recurrir a otros instrumentos de tutela como el derecho marcario.

Debe tenerse presente que el diseño de moda por naturaleza es cíclico, y que existe un sustento teórico que fomenta este ciclo. ${ }^{16}$ En este sentido, la dinámica del mercado hace que el ciclo de vida de un producto de moda sea tan corto que casi no merezca la pena tramitar formalmente una solicitud. Podría ser también que una empresa lance simultáneamente varias líneas de productos, sin tener la certeza de cuales van a ser los que perduren en el tiempo por tener mayor aceptación y cuales tendrá que abandonar. Para estos casos, existe la figura ya señalada en el derecho comunitario del diseño no registrado que permite obtener, por un periodo de tres años, la protección de un diseño que haya sido hecho accesible al público, sin tener que efectuar tramitación administrativa y estar sometido a un tiempo de protección innecesariamente largo en consideración con la naturaleza de la moda.

Sin embargo, cabe señalar que el diseño no registrado solamente permite impedir la copia no autorizada del mismo. Por lo tanto, hay que probar que ha existido acceso previo al producto y que se ha hecho un uso indebido. Si un tercero llegara a un resultado idéntico por sus propios medios, no se podrá hacer valer en ningún caso la protección ofrecida al diseño no registrado. Esta situación es diferente cuando se presenta una solicitud formal de diseño, ya que en este caso bastará que exista un parecido suficiente, independientemente de que haya copia o sólo una eventual coincidencia. El documento presentado puede hacerse valer frente a terceros.

Siendo así el estado de las cosas y teniendo en cuenta los plazos de la prioridad de exposición que conceden la legislación comunitaria y la respectiva la legislación nacional de cada uno de los países de la Unión Europea, corresponderá a cada empresa elegir la estrategia de protección que considere más conveniente en torno a su producción.

Es innegable que los mecanismos de protección de los diseños relativos a la indumentaria corresponderán finalmente a la legislación del país donde se reclama la tutela. Esta última determinará la vía adecuada para obtenerla, bien sea a través del sistema de propiedad industrial, mediante los derechos de autor, empleando ambos sistemas, o a través de mecanismos especiales.

En este sentido, somos de la opinión de que las formas clásicas de protección analizadas pudieran no ser suficientes en relación a la tecnología ponible.

\footnotetext{
${ }^{16}$ Raustiala, Kal y Sprigman, Christopher, "The piracy paradox: innovation and intellectual property in fashion design", Virginia Law Review, vol. 92. [Consulta: 10 de julio, 2017]. Disponible en: https://www.law.virginia.edu/pdf/faculty/ sprigman_piracy.pdf
} 
Con base en las consideraciones acotadas, se puede evidenciar que, por su naturaleza, la tecnología ponible involucraría no sólo una aparente plataforma sólida de innovaciones vinculadas al sistema de patentes, aparejadas al diseño industrial y al régimen marcario; sino que, al mismo tiempo, debe considerarse que nos encontramos ante un nuevo tipo de moda que también por su naturaleza está destinada a ser temporal y, por lógica consecuencia, a ser reemplazada por una nueva tendencia en fashion tech que irá de la mano con el avance tecnológico. Esto necesariamente significa que un modelo determinado quedaría desfasado en un breve periodo, probablemente menos de tres años, en la medida en que el modelo en mención no podría soportar un sistema tecnológico más avanzado, sea por la capacidad inicial con la que fue elaborado, por las funcionalidades inherentes con las que fue creado o por las compatibilidades del sistema que emplea.

Siendo así, consideramos que las diferentes modalidades adoptadas por las legislaciones mencionadas devendrían en insuficientes para proteger a la tecnología ponible debido a que, por las razones señaladas, esta figura sobrepasaría por mucho a los parámetros y condiciones de protección recogidos en las normas vigentes debido a las características que presenta.

\section{La tecnología ponible (wearable technology)}

La presencia de la tecnología cada vez más pequeña, inteligente, simplificadora e integrada a los artefactos que nos rodean en todos los campos del quehacer humano es indiscutible. Esto es así al grado que no sólo ha llegado a convertirse en una muestra de cierto estándar social, sino que también su uso ha logrado generar cierto grado de dependencia en todos nosotros, por lo que el mundo de la moda no podía sernos ajeno.

Steve Mann, ${ }^{17}$ experto en el tema, ha definido a la Wearable computer como el estudio o práctica de la invención, diseño, la construcción o el uso de dispositivos de cómputo y sensoriales que se adaptan a la anatomía del cuerpo humano. Los dispositivos se pueden llevar debajo, por encima o en la ropa o también pueden ser ellos mismos la ropa (smart clothing).

Según Mann, el campo de la computación vestible se extiende más allá de la ropa inteligente. Steve Mann utiliza el término Body-borne computing o Bearable computing como un sustituto para la computación vestible, para

\footnotetext{
${ }_{17}$ Steve Mann, profesor de la Universidad de Toronto, fue reconocido como "The Father of the Wearable Computer", y el fundador del campo de las tecnologias vestibles.
} 
incluir todas las formas de tecnología que están sobre o dentro del cuerpo. Un ejemplo podrían ser los teléfonos inteligentes dentro de sector de los dispositivos portátiles. ${ }^{18}$

En este contexto, tratar de dirigir la tutela jurídica a este nuevo tipo de tecnología obliga retomar los temas planteados anteriormente. Siendo así, tradicionalmente han existido marcadas diferencias entre las figuras conformantes de la propiedad intelectual, a pesar de la existencia de zonas fronterizas, a las cuales hemos hecho referencia. Sin embargo, la presencia de la tecnología ponible o vestible parece plantearnos nuevos cuestionamientos que nos conducirían esta vez a una zona de arenas movedizas en torno al tema.

En algún momento, Steve Jobs señaló que algunas personas creen que el diseño es el aspecto, pero cuando se profundiza podemos darnos cuenta que en realidad es el cómo funciona y que indiscutiblemente la omisión de la forma con función se evidencia en la interrelación e interacción que se produce entre la tecnología y la moda. De aceptar esta afirmación, estaríamos desvirtuando la naturaleza del diseño y subsumiendo el binomio tecnología y moda tan sólo al sistema de patentes.

Lo anterior significaría negar la importancia que ha logrado desarrollar el diseño en los productos, el cual ha trascendido al extremo de convertirse en uno de los factores determinantes para su adquisición. Los envases, envoltorios y formas que determinan su apariencia exterior y conceden a los ojos del consumidor un valor añadido poseen un invaluable valor económico, al punto de que se ha llegado a decir que el envase vende el producto.

Incluso este valor añadido que poseen algunos productos es especialmente evidente en aquellos que cada temporada sufren una profunda renovación. Se trata de productos de corta vida comercial, que una vez transcurrido el periodo para el que fueron ideados pierden su valor y son sustituidos por otros. Dicha situación bien podría aplicarse a la tecnología ponible.

El mercado tecnológico actual, mediante sus diversos e innovadores productos, ofrece infinidad de posibilidades en busca de elevar el nivel de bienestar o brindar comodidad a sus usuarios, al tiempo que crea un valor exponencial para las empresas. Sin embargo, su futuro aún no está decidido.

Por otro lado, debe tenerse presente que, además de los cuestionamientos abordados en torno a la propiedad intelectual, esta tecnología ponible enfrenta algunos retos que van desde un total nivel de aceptación por los usuarios, el

\footnotetext{
${ }^{18}$ De hecho, la palabra portátil viene de la palabra en francés porter, que significa to wear en inglés o vestir en español.
} 
poder adquisitivo de éstos, la duración de la batería de los dispositivos, hasta su capacidad de compatibilidad, conexión o interoperabilidad. Al mismo tiempo, genera preocupación en el ámbito jurídico, ya que las implicancias de su uso nos llevan a cuestionarnos sobre el manejo y control de los datos personales obtenidos a través de esta tecnología, la invasión de la privacidad de los usuarios, y la seguridad personal.

Adicionalmente, no podemos dejar de mencionar la presencia de la tecnología invasiva, que si bien permitirá potencializar las capacidades humanas (al hacer que los dispositivos formen parte de nuestro cuerpo de manera permanente), también generará nuevas modalidades directas de interacción con diversos dispositivos a nuestro alrededor y, en consecuencia, surgirán nuevos problemas para el derecho.

Asimismo, Poole, ${ }^{19}$ analista ejecutiva de la oMPI, considera que el sector de la tecnología ponible se encuentra en su etapa incipiente. Esto por el hecho de que en la actualidad se está desarrollando una amplia variedad de productos con esta tecnología. Si bien muchos están ya en el mercado, otros aún son sólo prototipos.

Es claro que nos encontramos ante productos que por su naturaleza congregan al sistema de patentes, al diseño, a la propiedad intelectual u otros. Pero al mismo tiempo, al estar inmersos dentro del sector de la moda, llevan consigo las características inherentes a ésta. Es decir, son productos cíclicos, de cambio constante y vida efímera dentro del mercado, como no lo son necesariamente las invenciones puras, los diseños industriales o las obras de arte aplicado, ya que por su propia naturaleza están destinados a tener un tiempo de duración relativamente largo. Esto se evidencia en los años de protección que conceden los ordenamientos jurídicos, con las respectivas posibilidades de renovación bajo el cumplimiento de determinados requisitos.

\section{Consideraciones finales}

La tecnología ponible está en constante cambio. Por esa razón, las innovaciones últimas desplazaran a las anteriores como lógico resultado del proceso de evolución tecnológica: las nuevas son mucho más completas, funcionales, llamativas, compatibles o simplemente portadoras de cierto estatus para sus usuarios.

\footnotetext{
${ }_{19}$ Poole, Emma, "The Brave New World of Wearable Technology: What Implications for IP?" Wipo Magazine, vol. 3. [Consulta: 12 de julio, 2017]. Disponible en: http://www.wipo.int/wipo_magazine/en/2014/03/article_0002.html
} 
Este hecho se traduce en una imparable competencia entre las grandes empresas con miras a producir la mejor tecnología ponible, que además de implicar una obligada renovación constante de sus productos (que lógicamente lleva a una existencia de corta duración de los mismos), genera gran demanda de los consumidores por conseguir la última moda que ofrece el mercado tecnológico. Este comportamiento consolida la vida efímera de la tecnología ponible dentro del mismo.

En este contexto, el sistema de protección actual en torno a este tipo de tecnología no está definido.

El presente estudio ha buscado analizar separadamente las figuras que están inmersas en la tecnología ponible dentro del marco de la propiedad industrial, los derechos de autor o los sistemas sui generis. La finalidad es esclarecer que nos encontramos ante una figura que, por su propia naturaleza y características, no logra encajar plenamente en ningún mecanismo de protección tradicional existente. Más aún, debido precisamente a sus condiciones inherentes y a la falta de consenso normativo entre moda y tecnología, podría en algún momento llegar a desnaturalizarse el diseño industrial ante la posibilidad de aceptar el hecho de que el diseño no es el aspecto, sino cómo funciona. En este sentido, en la relación moda y tecnología se evidenciaría la omisión de la forma con función.

Consideramos que el desfase entre la normativa actual y la tecnología ponible demanda un estudio profundo y especializado conducente a contar con un mecanismo de protección eficaz y eficiente acorde con su naturaleza. Más aún si asumimos el innegable hecho de que no sólo ha llegado para quedarse entre nosotros, sino que su crecimiento y uso se ha vuelto exponencial. 


\section{Bibliografía}

“35 U.S. Code $\$ 171$ - Patents for designs”, Legal Information Institute. [Consulta: 2 de julio, 2017]. Disponible en: https://www.law.cornell.edu/uscode/ text/35/171

"Acuerdo sobre los aspectos de los Derechos de Propiedad Intelectual relacionados con el comercio", World Trade Organization. [Consulta: 5 de julio, 2017]. Disponible en: https://www.wto.org/spanish/docs_s/legal_s/27-trips.pdf

"Convenio de Berna para la protección de las obras literarias y artísticas", Organización Mundial de la Propiedad Intelectual. [Consulta: 2 de julio, 2017]. Disponible en: http://www.wipo.int/treaties/es/text.jsp?file id=283698

"Convenio de París para la Protección de la Propiedad Industrial”, Organización Mundial de la Propiedad Intelectual. [Consulta: 2 de julio, 2017]. Disponible en: http://www.wipo.int/wipolex/es/treaties/text.jsp?file_id=287557

"Copyright law of the United States", Copyrigth.gov. [Consulta: 10 de julio, 2017]. Disponible en: https://www.copyright.gov/title17/title17.pdf

ERner, Guillaume, Sociología de las tendencias, Barcelona, Gustavo Gili, 2013.

“Ley sobre el Derecho de Autor”, Diario Oficial El Peruano, 24 de abril, 1996.

Poole, Emma, "The Brave New World of Wearable Technology: What Implications for IP?" Wipo Magazine, vol. 3. [Consulta: 12 de julio, 2017]. Disponible en: http://www.wipo.int/wipo_magazine/en/2014/03/article_0002.html

Raustiala, Kal y Sprigman, Christopher, "The piracy paradox: innovation and intellectual property in fashion design", Virginia Law Review, vol. 92. [Consulta: 10 de julio, 2017]. Disponible en: https://www.law.virginia.edu/pdf/faculty/ sprigman_piracy.pdf

Salas Pasuy, Brenda, "La industria de la moda a la luz de la propiedad intelectual”, La Propiedad Inmateria, vol. 17. [Fecha de consulta 1 julio 2017]. Disponible en: http://revistas.uexternado.edu.co/index.php/propin/article/ view/3583/3800 